\title{
Chile en el camino al Abolicionismo El siguiente paso
}

AUTOR: Dr. Antonio Muñoz Aunión.

En el año 2001, la República de Chile abolió la pena capital de sus sistemas de penas ordinarias por Ley n.19.734, el pasado mes de abril se cumplieron 13 años de esta importante fecha para la sociedad chilena y para la Comunidad internacional en su conjunto dado que siete años más tarde su compromiso con el Derecho internacional y el abolicionismo se acentúo con la ratificación del Segundo Protocolo facultativo de 1989 al Pacto Internacional de Derechos civiles y políticos, y en el ámbito regional, del Protocolo al Convenio americano sobre derechos humanos relativo a la abolición de la pena de muerte de 1990, convirtiéndose de esta forma en el País número ciento y ocho que lo hacía.

Estas fechas marcan un parteaguas, y desde entonces más y más Estados han engrosado este selecto club de países llamados en térmi- nos generales abolicionistas que consideran esta forma de pena una afrenta a la dignidad humana y contraria a los principios de la moderna ciencia penal y criminológica. Este grupo de Estados que comparten estos valores representan un relevante elemento de presión no sólo por su peso político sino también económico, según datos de la recién creada Comisión Internacional contra la pena de muerte apoyándose en informes de Naciones Unidas, más de 150 países han abolido la pena capital, bien de iure o de facto. (Hood, R.; " Staying Optimistic " en Capital Punishment. A Hazard to a Sustainable Criminal Justice System. ? "p. 305 (ed. Lill Scherdin, ASHGATE 2013)

Dentro del disminuido y heterogéneo grupo de Estados retencionistas se encuentran, entre otros, Irán, China, Japón, Arabia Saudita, y ciertos estados de los Estados Unidos.

A través de una práctica concertada del primer grupo, la actividad de distintas Organizaciones no gubernamentales, y de las votaciones en el seno de las Instituciones internacionales será posible mantener la inercia del abolicionismo. 
De hecho, los expertos más optimistas, con la cautela que toda predicción debe tener en el ámbito político y social, hablan de una fecha tentativa en la que desaparecerá de la faz de la Tierra, que se produciría en la tercera década de este siglo, augurio que contrasta claramente con el pesimismo de décadas precedentes (Newsletter, Oficina de Naciones Unidas para la prevención del delito y la justicia penal, 1986, "parece improbable que el objetivo de abolición de la pena capital pueda alcanzarse en algún momento .")

No obstante, y pese a que los indicadores son en todo caso halagüeños, no se puede caer en la autocomplacencia y pensar que con la eliminación de esta pena, el valor de la dignidad humana está completamente asegurado, sírvase pensar en la figura de los asesinatos selectivos, ejecuciones extrajudiciales, situación de hacinamiento y privación en el cumplimiento de la pena, es decir otras formas crueles de acaecimiento de la muerte bajo la autoridad estatal siguen presentes y es muestra de que el camino aún queda lejos de estar expedito.

Con ocasión de este feliz aniversario y aprovechando que la República de Chile ostenta un espacio privilegiado durante los próximos dos años en el Consejo de Seguridad sería deseable promover la ratificación de los textos que piden la abolición de "la pena innoble" en todo tiempo y circunstancia, esto implicaría un esfuerzo noble para el gobierno chileno que se mostraría a su vez dispuesto a renunciar a las reservas que permiten la pena capital para delitos especialmente graves en la Jurisdicción militar, y que por definición significaría cruzar un Rubicón imaginario hacia el terreno de la humanización de las normas penales. ${ }^{1}$

La abolición de la pena de muerte en el Código penal militar es una obligación tanto jurídica como moral y se encuentra también relacionada con factores estadísticos, la pervivencia de esta pena como castigo draconiano bien para aquellas personas que se encuentran bajo la aplicación de este fuero, o para aquellos que trasgreden los valores que ampara la Institución militar es puramente anecdótica incluso discriminatoria, si fijamos la vista atrás, en 1985 se aplicó esta pena por fusilamiento a dos miembros de Carabineros de Chile por su participación en unos macabros asesinatos, en un proceso que aún presenta graves interrogantes. ${ }^{2}$

${ }^{1}$ En el sentido del artículo 27 de la Constitución italiana que establece: " las penas no podrán consistir en tratos contrarios al sentido de humanidad ...

${ }^{2}$ Un siglo atrás la irracionalidad de la pena y la ausencia de imparcialidad se hace aún más evidente en 
Esta pervivencia en Chile que recordemos sólo se aplicaría en el hipotético caso de un conflicto militar declarado, ${ }^{3}$ la diferencia de otros países de Latinoamérica que han pasado a formar parte de la ilustre lista de abolicionistas en todo tiempo y circunstancia, por ejemplo, la reciente adhesión de Bolivia y Argentina sin reservas al Segundo Protocolo del Pacto Internacional de Derechos Civiles y Políticos, ${ }^{4}$ implica un proceso de normalidad difusa por cuanto a su papel destacado como defensora de los derechos humanos tanto en su jurisdicción como en el exterior.

Se trata de esbozar un mundo ideal donde sea más probable la muerte por un objeto caído del espacio que mediante cualquier forma de asesinato legal o muerte estatal, el hecho de mantener esta forma de pena resulta equiparable por cuanto a sus efectos de incertidumbre a los denominados asesinatos selectivos, linchamientos populares, o ejecuciones extrajudiciales todas ellas censurables y que hoy día repuntan bien por los avances tecnológicos que permiten acabar con la vida del " designado " desde la distancia, o por la desidia en la gestión del control social bien descentralizado mediante una concienciación y educación de la sociedad civil, o regulado a través de la separación de poderes. El hecho de poder acercar la muerte sigilosamente desde la distancia a aquellos señalados y con control absoluto de su destino a través de verdaderas máquinas de matar, que fungen como verdugos en de un siglo marcado por la tecnología, es un insulto

\footnotetext{
el caso del enjuiciamiento por un Consejo de Guerra y posterior fusilamiento del político porteño Ricardo Cunning a resultas del convulso período revolucionario y cuyo destino hubiera sido bien distinto si la captura se hubiera producido a tiempo con el advenimiento y toma del poder por el movimiento Congresista.

En distinta latitud, cabe mencionar la ejecución de Mary Surratt, primera mujer en ser ejecutada en Estados Unidos por su implicación en el asesinato del Presidente Lincoln que fue producto de una situación y proceso en circunstancias excepcionales y con la aplicación de unas reglas procesales sobre participación en actividad delictiva mucho más débiles que las de hoy en día, en más detalle, Kauffman, M.; American Brutus.

${ }^{3}$ Hoy día la cuestión de la declaración de guerra no tiene mayor relevancia al ser básicamente una cuestión de hecho, la existencia de un conflicto armado de intensidad y bandos enfrentados con organización, extensible mutatis mutandis a conflictos internos. Cfr.; ICTY, Fiscal c. Tadic No. IT 94-1- T Decisión sobre la excepción de Falta de Jurisdicción de 1 de octubre 1995 \# 70.

${ }^{4}$ Consecuencia lógica de su mandato constitucional aprobada en 2009 cuyo artículo 15 párrafo 1 declara: " "Toda persona tiene derecho a la vida y a la integridad física, psicológica y sexual. Nadie será torturado, ni sufrirá tratos crueles, inhumanos, degradantes o humillantes. No existe la pena de muerte". Cursivas del autor.
} 
a la dignidad humana y una espada de Damocles para las víctimas que pueden inclusive, no saber cuál es su rol en todo el entramado de la política internacional o nacional, y hacer a algunos sentirse omnipotentes rompiendo el credo de la igualdad de todos los hombres y la propia igualdad soberana de los Estados mucho más peligroso que matanzas a escala global que siempre tienen más instancias de control y en caso de cometerse existen mecanismos para determinar la responsabilidad de los participantes.

Si el abolicionismo trata de limitar los daños directos y colaterales que la pena de muerte implica, el paso subsiguiente es acabar con ella en el terreno militar alcanzándose así la teoría del riesgo cero, y la consolidación de la dignidad humana ${ }^{5}$ y atenuación de la crueldad humana. Este argumento de la crueldad no es baladí y supone una vía por la que numerosos países están poniendo fin a la máxima pena, a través de lo que se conoce como el "estándar evolutivo " dado que tanto durante el proceso, la reclusión, como la ejecución es improbable llegar a alcanzar un umbral de crueldad "aceptable." 6

Otro argumento de peso, de naturaleza científica, criticado por los abolicionistas per se, se encuentra en la propia falibilidad del proceso penal que se acentúa en el terreno militar como muestra la discordante Jurisprudencia nacional e internacional en la materia desde Núremberg y en la actualidad de interés para el Derecho penal económico, por cuanto a la cuestión de la responsabilidad criminal, a saber, cuestiones referentes a la participación, en concreto las tesis sobre autoría mediata, la responsabilidad de mando y la obediencia debida; ${ }^{7}$ espacios donde la tarea de juzgar se torna pantanosa y la

\footnotetext{
${ }^{5}$ En el Reino Unido, la " dignidad humana " fue el germen que inspiro una transformación a través de la Ley, que llevo a Engels a afirmar que el Parlamento Británico era el organismo más revolucionario de Europa, cfr, Capps, P.; Human Dignity and the Foundations of International Law, Oxford Hart Publishing 2009, p. 201

${ }^{6}$ En este sentido cabe destacar, el artículo 5 de la Declaración Universal de los derechos del hombre de 1948, y en el contexto regional, el artículo 5. 2 del Pacto de San José: "Nadie debe ser sometido a torturas ni a penas o tratos crueles, inhumanos degradantes.

En la actualidad, ninguna forma de ejecutar se ha comprobado que sea indolora o incruenta, y las técnicas de combinación de cocktails de la muerte no producen la pretendida muerte dulce como se ha puesto de relieve en la última ejecución fallida que ha culminado en una dantesca imagen de sufrimiento humano. Cfr, Arroyo Zapatero, L.; Hood, R.; "Pena de muerte: Abolición “ Revista de Occidente junio 2013 p. 15

${ }^{7} \mathrm{Cfr}$, con la Resolución II. 1 de la Sesión I del XVI Congreso de la Asociación Internacional de Derecho Penal (AIDP) Budapest 1999 que recalcaba la dificultad y adelantaba una solución que suscita gran
} 
cuestión probatoria se vuelve difusa por mor de razones espaciales, temporales, personales y políticas que llevan a la aplicación de esta pena a una suerte de ruleta rusa impulsada por dos modelos distintos de alcanzar la Justicia, el sistema anglosajón vs., el continental y las contradicciones en sus mismos fueros, ${ }^{8}$ y todo esto, a pesar de los intentos de codificación y su híbridización progresiva por la Jurisprudencia y el Estatuto de Roma, no obstante la claridad meridiana del art. 75.4 b) del Protocolo I adicional de Ginebra. ${ }^{9}$

Es por estos motivos, que desde este foro académico se pide a las Autoridades competentes que inicien los trámites legales necesarios para denunciar la reserva, con ocasión de que este año se cumple el vigésimo quinto aniversario de su entrada en vigor, este paso del ecuador tiene una gran significancia a sabiendas de que los plazos temporales no son de igual aplicación a las cuestiones de jurisdicción militar inextricablemente unidas a la noción clásica de soberanía. ${ }^{10}$

A esto hay que añadir, la débil legitimidad normativa, ${ }^{11}$ la menor eficacia de las salvaguardias, ${ }^{12}$ y a su deriva inflacionista en tiempos de guerra declarada o no, en el pasado se encuentra en la lista de crímenes sujetos a la pena capital en Alemania en los albores de la Segunda guerra mundial que ascendían a 3 y que pasaron a 43 poco antes de

\footnotetext{
incertidumbre respecto de la aplicación irresoluble de la pena capital; “ En razón de que a menudo es difícil probar que los líderes y los miembros de grupos criminales organizados han efectivamente participado en la comisión de ciertos delitos, las formas tradicionales de comisión y la responsabilidad penal accesoria pueden ser insuficientes para hacer que estos individuos respondan penalmente. En cuanto a las categorías del derecho tradicional de autoría y complicidad juzgadas como insuficientes, se debería considerar una cautelosa modernización basada sobre el principio de responsabilidad organizacional. En las organizaciones estructuradas jerárquicamente, las personas con capacidad de decisión y control pueden ser responsables por actos de otros miembros bajo su control si ellos han ordenado que estos actos sean cometidos o han omitido conscientemente impedir su comisión.

${ }^{8} \mathrm{Cfr}$. Con los asuntos Yamashita, My Lai, y otros

9" Nadie podrá ser condenado por una infracción si no es sobre la base de la responsabilidad penal individual.

${ }^{10} \mathrm{Cfr}$, la obra maestra del cine del siglo XX de Stanley Kubrick Senderos de Gloria y su mensaje pacifista y contrario a la pena capital en razón de sus fines espurios, cuya exhibición en sala fue prohibida durante más de dos décadas por países con democracias bien asentadas, a saber, Estados Unidos, Francia y otros.

${ }^{11}$ Así el encaje de los delitos en los diferentes artículos del Código de Justicia Militar permite arbitrariedades con poco riesgo de recusación.

${ }^{12}$ Schabas, W.; The Abolition of the death penalty in International Law Cambridge University Press 3 ed. 2002 p. 211
} 
concluir esta, ${ }^{13}$ más recientemente, en la ausencia de garantías procesales debidas en los Estados Unidos respecto de los denominados " combatientes enemigos " en la lucha contra el Terror. Finalmente, la gran teatralidad que implica el sistema procesal militar: un edificio gris y esquivo al escrutinio público, llenos de salas y fríos corredores, los participantes en el Tribunal con sus uniformes de las distintas armas, una iluminación ad hoc digna de una buena obra de terror, y en última instancia, a la espera de conocer su destino en un complejo sistema procesal penal que desdeña su dignidad, el acusado.

Con seguridad, estos pasos llevaran a desterrar esta cruel calamidad del continente americano estrechando el cerco y haciendo realmente peculiar y dramática su pervivencia residual en ciertos estados de la Unión americana, uniéndose al destino de Europa que se configuró como el primer espacio libre de pena capital, ${ }^{14}$ asimismo con una notable excepción retencionista, Bielorrusia. Indefectiblemente, será imposible por propios cálculos estadísticos proteger a la Humanidad en su conjunto al 100 por 100 pero el éxito de la gobernanza mundial podrá certificarse cuanto más próxima de esta cifra se encuentren los datos, y cuando las probabilidades de morir ejecutado, en el continente asiático y africano sean menores que las de ser alcanzado por un meteorito que atraviese las capas atmosféricas de nuestro planeta.

La consecuencia de esta medida y de la cadena de Protocolos, a través de una política que vincule la firma y ratificación de los mismos como una parte esencial de la negociación de acuerdos con terceros países, a corto plazo sería crear un cordón sanitario y de aislamiento frente a estos pocos Estados recalcitrantes que situaría a la muerte como un fracaso de todo sistema de Derecho penal y sería coherente con las estadísticas que muestran que en la mayoría de las sociedades contemporáneas se empuja a la muerte a la derrota en los más diversos escenarios, a saber, descenso de la mortalidad infantil, mejora de la salud, prolongación de la vida, descenso de la tasa de homicidios, reducción de las muertes de tráfico, desaparición de la pena de

\footnotetext{
${ }^{13}$ Gellately, R.; Backing Hitler: Consent and Coertion in Nazi Germany, Oxford University Press 2002.-

${ }^{14}$ Cabe destacar que esta es una característica común a todo ciudadano de la U.E, sus vidas e integridad física quedan amparadas en razón de que el territorio de la Unión Europea es un espacio libre de pena capital y se respeta el principio de non- refoulement. Con el ánimo de dar mayor credibilidad a este espacio, el recientemente creado Servicio Europeo de Acción Exterior al servicio de la Unión protege a los ciudadanos a través de la protección diplomática y la adopción de posiciones comunes en el marco de la Política Exterior y de Seguridad Común.
} 
muerte para delitos comunes, lo que ejemplifica que una gran parte de la Humanidad corre menos riesgos a medida que avanza el siglo, es por ello lógico que los códigos penales militares hagan desaparecer esta pena cruenta de sus páginas. 


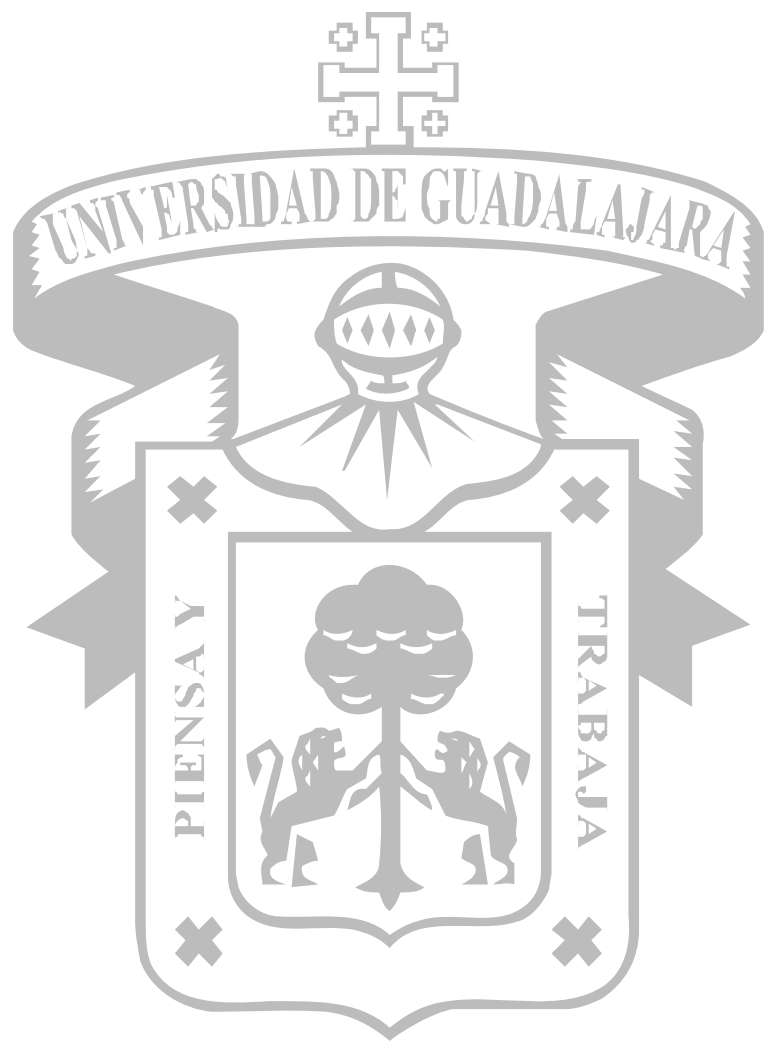




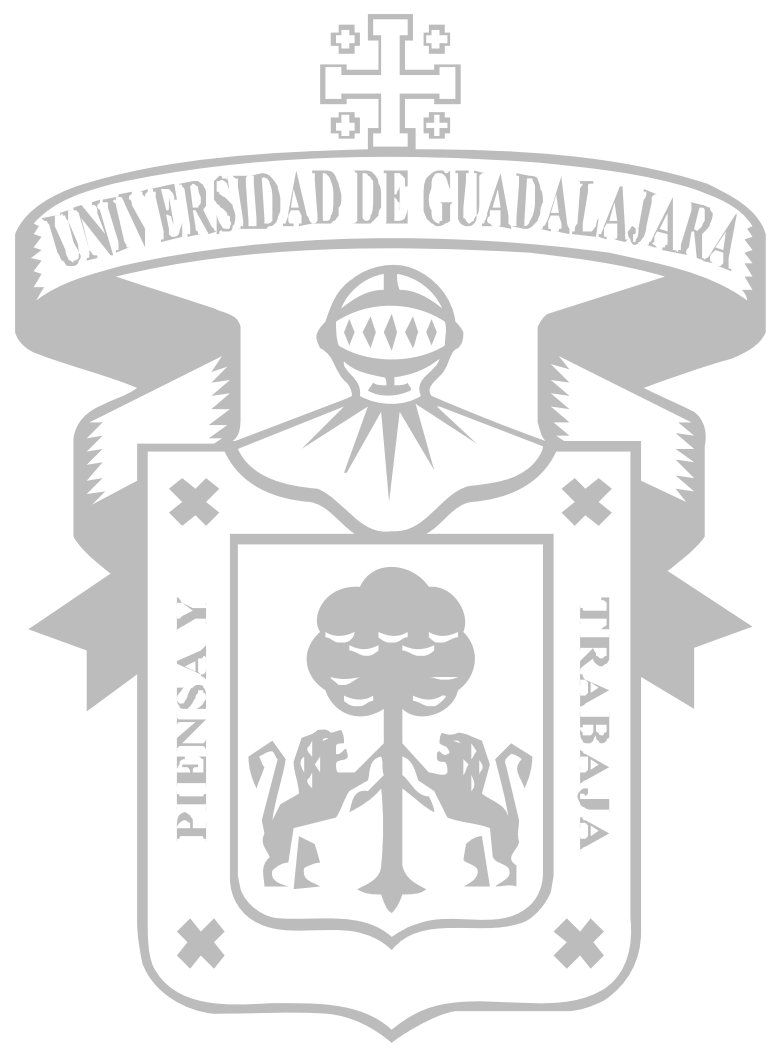

